\title{
RATE OF CONVERGENCE TO THE ROSENBLATT DISTRIBUTION FOR ADDITIVE FUNCTIONALS OF STOCHASTIC PROCESSES WITH LONG-RANGE DEPENDENCE ${ }^{1}$
}

\author{
N.N. LEONENKO \\ Kyiv University (National) \\ Department of Mathematics \\ Kyiv 252601, Ukraine \\ E-mail: leonenkon@Cardiff.ac.uk \\ V.V. ANH \\ Queensland University of Technology \\ School of Mathematical Sciences, GPO Box 2434 \\ Brisbane $Q$ 4001, Australia \\ E-mail: v.anh@fsc.qut.edu.au
}

(Received October, 1999; Revised October, 2000)

This paper establishes the rate of convergence (in the uniform Kolmogorov distance) for normalized additive functionals of stochastic processes with long-range dependence to a limiting Rosenblatt distribution.

Key words: Nonlinear Functionals, Gaussian Processes, Long-Range Dependence, Rosenblatt Distribution, Rate of Convergence.

AMS subject classifications: $62 \mathrm{E} 20,60 \mathrm{~F} 05$.

\section{Introduction}

The study of random processes and fields with correlations decaying at hyperbolic rates, i.e., those with long-range dependence (LRD), presents interesting and challenging probabilistic as well as statistical problems. Progress has been made in the past two decades or so on the theoretical aspects of the subject. On the other hand, recent applications have confirmed that data in a large number of fields (including hydrology, geophysics, turbulence, economics and finance) display LRD. Many stochastic models have been developed for description and analysis of this phenomenon. For recent developments, see Beren [6], Barndorff-Nielsen [5], Anh and Heyde [2], Leonenko

\footnotetext{
${ }^{1}$ The work was partially supported by the Australian Research Council grant A69804041 and NATO grant PST.CLG.976361.
} 
[20], among others.

The non-central limit theorem, which describes the limiting distributions of additive functionals, plays a key role in the theory of random processes and fields with LRD. The main references here are Taqqu [35, 37], and Dobrushin and Major [10] (see also, Surgailis [34], Samorodnitsky and Taqqu [33], Leonenko and Šilac-Benšic [21], Ho and Hsing [16], Leonenko and Woyczynski [24], Anh and Leonenko [4], among others). The limiting distributions have finite second-order moment but may have non-Gaussian structure. The problem of rate of convergence in the non-central limit theorem is therefore of considerable interest.

Some results on the rate of convergence to the Gaussian distribution for integral functionals of Gaussian random processes and fields with LRD were considered by Leonenko [19] (see also, Ivanov and Leonenko [17], pp. 64-70, Leonenko et al. [22], and Leonenko and Woyczynski [23]). These results correspond to Hermitian rank $m=1$ (defined in Section 2).

In this paper, we provide the rate of convergence (in the uniform Kolmogorov distance) of probability distributions of normalized integral functionals of Gaussian processes with LRD and a special form of the covariance function (see condition $\mathrm{C}$ below) to a limiting non-Gaussian distribution called the Rosenblatt distribution. The result corresponds to Hermitian rank $m=2$, which is new.

\section{Preliminaries}

Let $(\Omega, \mathcal{F}, P)$ be a complete probability space and $\xi(t)=\xi(\omega, t): \Omega \times \mathbb{R} \rightarrow \mathbb{R}$ be a random process in continuous time.

We first list the relevant assumptions, not all of which will be needed at the same time.

A. The process $\xi(t), t \in \mathbb{R}$, is a real measurable mean-square continuous stationary Gaussian process with mean $E \xi(t)=0$ and covariance function $B(t)=B(|t|)=\operatorname{cov}(\xi(0), \xi(t)), t \in \mathbb{R}$, such that $B(0)=1$.

$\mathrm{A}^{\prime}$. The covariance function $B(t), t \in \mathbb{R}$, is of the form

$$
B(t)=\frac{L(|t|)}{|t|^{\alpha}}, 0<\alpha<1
$$

where $L(t):(0, \infty) \rightarrow(0, \infty)$ is bounded on each finite interval and slowly varying for large values of $t$; i.e., for each $\lambda>0, \lim _{t \rightarrow \infty}[L(\lambda t) ; L(t)]=1$.

Most of the papers devoted to limit theorems for random processes with LRD have used the covariance function of the form (1). Nevertheless, for continuous-time processes, it is not easy to find exact examples of non-negative definite continuous functions which satisfy (1). Note that the class of covariance functions of real-valued stationary processes coincides with the class of characteristic functions of symmetric probability distributions. From the theory of characteristic functions we are currently able to present only the following examples of covariance functions of the form (1):

$$
\begin{gathered}
B_{0}(t)=\left(1+t^{2}\right)^{-\alpha / 2}, \quad 0<\alpha<1 ; \\
B_{1}(t)=\left(1+|t|^{\alpha}\right)^{-1}, \quad 0<\alpha<1 ; \\
B_{2}(t)=\left(1+|t|^{\beta}\right)^{-\nu}, \quad 0<\beta \nu<1, t \in \mathbb{R} .
\end{gathered}
$$


The function $B_{0}(t), t \in \mathbb{R}$, is known as the Fourier transform of Bessel potential (see Donoghue [11], p. 294) or characteristic function of symmetric Bessel distributions (see Oberhettinger [27], p. 156, or Fang et al. [14], p. 69). It has the spectral representation

$$
B_{0}(t)=\int_{\mathbb{R}} \cos (\lambda t) f_{\alpha}(\lambda) d \lambda
$$

with an exact form of spectral density $f_{\alpha}$ (see (17) below) such that

$$
f_{\alpha}(\lambda) \sim c_{1}(\alpha)|\lambda|^{\alpha-1}, \quad 0<\alpha<1,
$$

as $\lambda \rightarrow 0$, where the Tauberian constant

$$
c_{1}(\alpha)=\frac{1}{2 \Gamma(\alpha) \cos (\alpha \pi / 2)}
$$

The process $\xi(t), t \in \mathbb{R}$, itself satisfying condition A with covariance function $B_{0}$, has the spectral representation

$$
\xi(t)=\int_{\mathbb{R}} e^{i t \lambda} \sqrt{f_{\alpha}(\lambda)} W(d \lambda),
$$

where $W(\cdot)$ is the complex Gaussian white noise.

The function $B_{1}(t), t \in \mathbb{R}$, is known as the characteristic function of the Linnik distribution (see Kotz et al. [18]). This distribution has a density function (i.e., the covariance function $B_{1}$ has a spectral density). Kotz et al. [18] investigated the asymptotic behavior at frequency 0 are quite distinct in the cases:

(i) $1 / \alpha$ being an integer,

(ii) $1 / \alpha$ being a non-integer rational number, and

(iii) $\alpha$ being an irrational number.

Similar properties hold for the covariance function $B_{2}(t), t \in \mathbb{R}$, which is known as the characteristic function of the generalized Linnik distribution (see Erdoğan and Ostrovskii [13]).

In this paper, we shall consider the covariance function $B_{0}(t), t \in \mathbb{R}$, as the key example of covariance functions of random processes in continuous time in the sense of representation (1). In principle, our method is also applicable to the cases of covariance functions $B_{1}(t)$ and $B_{2}(t)$. The method uses the second term in the asymptotic expansion of the spectral density at frequency zero, which depends on the arithmetic nature of the parameter $\alpha$ (see Kotz et al. [18]). Hence the rate of convergence depends on the arithmetic nature of $\alpha$ and is different for the cases $(i)-(i i i)$. This problem will be addressed elsewhere.

Viano et al. [39] introduced continuous-time fractional ARMA processes. Some asymptotic results for the correlation functions and spectral densities of these processes were obtained. However, these results are not useful to the problem of this paper, since in our approach we need exact results (such as Lemma 4.5 below) on the asymptotic behavior of the spectral density at frequency zero. See also Remark 3.3 below.

B. A non-random Borel function $G: \mathbb{R} \rightarrow \mathbb{R}$ is defined such that 
with

$$
\int_{-\infty}^{\infty} G^{2}(u) \varphi(u) d u<\infty
$$

$$
\varphi(u)=\frac{1}{\sqrt{2 \pi}} e^{-u^{2} / 2}, u \in \mathbb{R}
$$

The nonlinear function $G(u), u \in \mathbb{R}$ can then be expanded in the series

$$
G(u)=\sum_{k=0}^{\infty} C_{k} H_{k}(u) / k !, C_{k}=\int_{-\infty}^{\infty} G(u) H_{k}(u) \varphi(u) d u, k=0,1,2, \ldots
$$

of orthogonal Chebyshev-Hermite polynomials

$$
H_{k}(u)=(-1)^{k} e^{u^{2} / 2} \frac{d^{k}}{d u^{k}} e^{-u^{2} / 2}, \quad k=0,1,2, \ldots,
$$

which form a complete orthogonal system in Hilbert space $L^{2}(\mathbb{R}, \varphi(u) d u)$.

Additionally, we will assume that the function $G$ satisfies the condition

$\mathrm{B}^{\prime}$. There exists an integer $m \geq 1$ such that $C_{1}=\ldots=C_{m-1}=0, C_{m} \neq 0$.

The integer $m \geq 1$ will be called the Hermitian rank $G$ (see, for example, Taqqu $[35,37]$; Dobrushin and Major [10]).

We state the following non-central limit theorem due to Taqqu [35, 37] and Dobrushin and Major [10]. See also Rosenblatt [32].

Theorem 2.1: Under conditions $A, A^{\prime}, B$ and $B^{\prime}$ with $\alpha \in(0,1 / m)$, where $m \geq 1$ is the Hermitian rank of the function $G$, the finite-dimensional distributions of the random processes

$$
Y_{T}(s)=\frac{1}{d(T)} \int_{0}^{T_{s}}\left[G(\xi(t))-C_{0}\right] d t, 0 \leq s \leq 1,
$$

with

$$
d(T)=T^{1-\alpha m / 2} L^{m / 2}(T)
$$

converge weakly, as $T \rightarrow \infty$, to the finite-dimensional distributions of the random process

$$
Y_{m}(s)=\frac{C_{m}}{m !}\left[c_{1}(\alpha)\right]^{m / 2} \int_{\mathbb{R}^{m}}^{\prime} \frac{e^{i\left(\lambda_{1}+\ldots+\lambda_{m}\right) s}-1}{i\left(\lambda_{1}+\ldots+\lambda_{m}\right)} \frac{W\left(d \lambda_{1}\right) \ldots W\left(d \lambda_{m}\right)}{\left|\lambda_{1} \ldots \lambda_{m}\right|^{(1-\alpha) / 2}}, \quad 0 \leq s \leq 1,
$$

where $C_{0}$ and $C_{m}$ are defined by (6) and $\int_{\mathbb{R}^{m}}^{\prime} \ldots$ is a multiple stochastic integral with respect to complex Gaussian white noise $W(\cdot)$ (with integration on the hyperplanes $\lambda_{i}= \pm \lambda_{j}, i, j=1, \ldots, m, i \neq j$, being excluded $)$.

Remark 2.1: The definition and properties of the multiple stochastic integral (8) can be found in Major [25] or Taqqu [37].

Remark 2.2: The normalizing factor $d(T)$ in (7) is chosen such that, as $T \rightarrow \infty$,

$$
\operatorname{var}\left[\int_{0}^{T} H_{m}(\xi(t)) d t\right]=d^{2}(T) m ! c_{2}(m, \alpha)(1+o(1))
$$


where

$$
c_{2}(m, \alpha)=\int_{0}^{1} \int_{0}^{1} \frac{d t d s}{|t-s|^{m \alpha}}=\frac{2}{(1-m \alpha)(2-m \alpha)}, 0<\alpha<\frac{1}{m} .
$$

Remark 2.3: Note that $E\left|Y_{m}(s)\right|^{2}<\infty$, but for $m \geq 2$ the process $Y_{m}(s)$ have non-Gaussian structure.

For a random process in continuous time, the proof of Theorem 2.1 may be constructed from Taqqu [37] and Dobrushin and Major [15] by using the argument of Berman [7].

The Gaussian process $Y_{1}(s), s>0$, defined in (8) with $m=1$, is fractional Brownian motion. This process plays an important role in applications in hydrology, turbulence, finance, etc. An extension of this process has been recently proposed by Anh et al. [3]. They introduced fractional Riesz-Bessel motion, which provides a generalization of fractional Brownian motion and describes long-range dependence as well as second-order intermittency. The latter is another important feature of turbulence and financial processes. The spectral density of increments of such processes is a generalization of the spectral density of fractional Ornstein-Uhlenbeck-type processes (see Comte [9]).

The process $Y_{2}(s), s>0$, defined in (8) with $m=2$, is called the Rosenblatt process (See Taqqu [35, 37]) because it first appeared in Rosenblatt [30] (see also Rosenblatt [31]). Some moment properties of these distributions can be found in Taqqu [35, 36] and Taqqu and Goldberg [38]. In particular, the marginal distribution of the random processes

$$
\begin{aligned}
& R_{2}(s)=Y_{2}(s)=\frac{C_{2}}{2} c_{1}(\alpha) \int_{\mathbb{R}^{2}}^{\prime} \frac{e^{i\left(\lambda_{1}+\lambda_{2}\right) s}-1}{i\left(\lambda_{1}+\lambda_{2}\right)} \frac{W\left(d \lambda_{1}\right) W\left(d \lambda_{2}\right)}{\left|\lambda_{1} \lambda_{2}\right|^{(1-\alpha) / 2}}, s>0 \\
& 0<\alpha<1 / 2
\end{aligned}
$$

is called the Rosenblatt distribution. Note that

$$
E R_{2}^{2}(1)=\left[\frac{C_{2}}{2} c_{1}(\alpha)\right]_{\mathbb{R}^{2}}^{2}\left|\frac{e^{i\left(\lambda_{1}+\lambda_{2}\right)}-1}{i\left(\lambda_{1}+\lambda_{2}\right)}\right|^{2} \frac{d \lambda_{1} d \lambda_{2}}{\left|\lambda_{1} \lambda_{2}\right|^{1-\alpha}}<\infty, 0<\alpha<1 / 2
$$

From Rosenblatt [30, 31], Taqqu [35] and Berman [7], we obtain the characteristic function of the random variable

$$
\overline{R_{2}}=R_{2}(1) /\left[C_{2} c_{1}(\alpha) / 2\right]
$$

It has the form

where

$$
E \exp \left\{i u \overline{R_{2}}\right\}=\exp \left\{-\frac{1}{2} \sum_{j=2}^{\infty} \frac{(2 i u)^{j}}{j} \aleph_{j}\right\}, u \in \mathbb{R}
$$

$$
\aleph_{j}=\int_{[0,1]^{j}} \frac{d x_{1} \ldots d x_{j}}{\left|x_{1}-x_{j}\right|^{\alpha} \prod_{k=2}^{j}\left|x_{k}-x_{k-1}\right|^{\alpha}}, 0<\alpha<1 / 2 .
$$

We shall also use the following representation of Rosenblatt distribution (12), which 
follows from the representation (10) and the results of McKean [26] (see also Dobrushin and Major [10]):

$$
\overline{R_{2}}=\sum_{k=1}^{\infty} \nu_{k}\left(X_{k}^{2}-1\right), \sum_{k=1}^{\infty} \nu_{k}^{2}<\infty, \sum_{k=1}^{\infty} \nu_{k}=\infty
$$

where $X_{k}, k=1,2,3, \ldots$, is a sequence of independent standard normal variables, and $\nu_{k}, \quad k=1,2,3, \ldots$, is a sequence of non-negative real numbers which are the eigenvalues of the self-adjoint Hilbert-Schmidt operator

$$
\mathcal{A} f(\lambda) \int_{\mathbb{R}} H\left(\lambda,-\lambda_{2}\right) f\left(\lambda_{2}\right) d \lambda_{2}: L_{2}(\mathbb{R}, d \lambda) \rightarrow L_{2}(\mathbb{R}, d \lambda)
$$

(see, for example, Dunford and Schwartz [12]) with the symmetric kernel

such that

$$
H\left(\lambda_{1}, \lambda_{2}\right)=\frac{e^{i\left(\lambda_{1}+\lambda_{2}\right)}-1}{i\left(\lambda_{1}+\lambda_{2}\right)}\left|\lambda_{1} \lambda_{2}\right|^{(\alpha-1) / 2}
$$

$$
H\left(\lambda_{1},-\lambda_{2}\right)=\overline{H\left(-\lambda_{1}, \lambda_{2}\right)} \text { and } \int_{\mathbb{R}^{2}}\left|H\left(\lambda_{1}, \lambda_{2}\right)\right|^{2} d \lambda_{1} d \lambda_{2}<\infty .
$$

Here $L_{2}(\mathbb{R}, d \lambda)$ is the Hilbert space of complex-valued functions $f(\lambda), \lambda \in \mathbb{R}$, such that

with scalar product

$$
f(\lambda)=\overline{f(-\lambda)}, \quad \int_{\mathbb{R}}|f(\lambda)|^{2} d \lambda<\infty
$$

$$
(f, g)=\int_{\mathbb{R}} f(\lambda) \overline{g(\lambda)} d \lambda .
$$

Let $\phi_{k}, k=1,2,3, \ldots$, be the complete orthonormal system of the eigenvectors of the operator $\mathcal{A}$. Then

$$
H\left(\lambda_{1},-\lambda_{2}\right)=\sum_{k=1}^{\infty} \nu_{k} \phi_{k}\left(\lambda_{1}\right) \overline{\phi_{k}\left(\lambda_{2}\right)}
$$

and by Itô's formula (see, for example, Taqqu [37])

$$
\begin{gathered}
\int_{\mathbb{R}^{2}}^{\prime} H\left(\lambda_{1}, \lambda_{2}\right) W\left(d \lambda_{1}\right) W\left(d \lambda_{2}\right)=\sum_{k=1}^{\infty} \nu_{k} H_{2}\left(\int \phi_{k}(\lambda) W(d \lambda)\right) \\
=\sum_{k=1}^{\infty} \nu_{k}\left(X_{k}^{2}-1\right),
\end{gathered}
$$

which can be traced back to McKean [26].

It is easy to see that $\mathcal{A}$ is a compact operator and the bounds

$$
m=\inf \{(\mathcal{A} f, f),\|f\|=1\}, M=\sup \{(\mathcal{A} f, f),\|f\|=1\}
$$

are different from zero; therefore they are in the spectrum of $\mathcal{A}$. Thus, there exist at 
least two non-zero eigenvalues $\nu_{p}$ and $\nu_{q}$ such that

$$
\beta=\frac{\nu_{p}}{\nu_{q}}<1
$$

In fact, at least one non-zero eigenvalue exists because $\mathcal{A}$ is a non-zero operator with non-zero norm. Suppose that there is only one non-zero eigenvalue $\nu_{1}$ with corresponding non-zero eigenvector $\phi_{1}(\lambda)$; then putting $\lambda_{1}=\lambda_{2}$ in (15) and using (11) we obtain $\lambda_{1}\left|\phi_{1}\left(\lambda_{1}\right)\right|^{2}=0$, which is a contradiction. Using the same argument, it is easy to prove that if there exist two non-zero eigenvalues, then they are different.

Recently, Albin [1] proved that the Rosenblatt distribution has a density function which belongs to the type-1 domain of attraction of extremes. Albin [1] also used the representation (13) where

$$
\nu_{1}=\ldots=\nu_{k_{0}}>\nu_{k_{0}+1} \geq \ldots>0
$$

and the Laplace transform of $\overline{R_{2}}$ is given by

for $s \in\left(-\left(2 \nu_{1}\right)^{-1}, \infty\right)$.

$$
E \exp \left\{-s \overline{R_{2}}\right\}=\exp \left\{-\sum_{j=1}^{\infty}\left(\frac{1}{2} \ln \left(1+2 \nu_{j} s\right)-\nu_{j} s\right)\right\}
$$

\section{Main Result}

We present a result on the rate of convergence (in the uniform Kolmogorov distance) of probability distributions of random variables $Y_{T}(1)$, defined in (7) for a special covariance function (see condition $\mathrm{C}$ below), to the Rosenblatt distribution of $R_{2}(1)$, defined in (10) or (12) and (13). Some results on the rate of convergence to the normal law along the line of Theorem 2.1 were obtained by Leonenko [19] (see also Ivanov and Leonenko [17, p. 64-70]). These results correspond to the case $m=1$ (see condition $B^{\prime}$ ) in Theorem 2.1. In this paper, we examine the case $m=2$.

For technical reasons, we formulate the following assumption for the covariance function.

C. The covariance function $B(t), t \in \mathbb{R}$, of the process $\xi(t), t \in \mathbb{R}$, has the form

$$
B(t)=\frac{1}{\left(1+t^{2}\right)^{\alpha / 2}}, 0<\alpha<1
$$

Remark 3.1: Let condition C hold. Then condition (1) is satisfied.

Remark 3.2: Under condition C, the spectral density $f(\lambda)=f(|\lambda|), \lambda \in \mathbb{R}$, has the following exact form (see, for example, Donoghue [11], p. 293, or Oberhettinger [27], p. 156):

$$
f_{\alpha}(\lambda)=f_{\alpha}(|\lambda|)=\frac{2^{(1-\alpha) / 2}}{\Gamma(\alpha / 2) \sqrt{\pi}} K_{(1-\alpha) / 2}(|\lambda|)|\lambda|^{(\alpha-1) / 2}, \lambda \in \mathbb{R},
$$

where

$$
K_{\nu}(z)=\frac{1}{2} \int_{0}^{\infty} s^{\nu-1} \exp \left\{-\frac{1}{2} z\left(s+\frac{1}{s}\right)\right\} d s, z>0
$$


is the modified Bessel function of the third kind of order $\nu$ (see, for example, Watson $[40])$. We note that

$$
K_{\nu}(z) \sim \Gamma(\nu) 2^{\nu-1} z^{-\nu}, z \downarrow 0, \nu>0
$$

and for a large value of $z$ the following approximation holds:

$$
K_{\nu}(z)=\sqrt{\frac{\pi}{2}} z^{-1 / 2} e^{-z}\left(1+\frac{\mu-1}{8 z}+\frac{(\mu-1)(\mu-9)}{2 !(8 z)^{2}}+\frac{(\mu-1)(\mu-9)(\mu-25)}{3 !(8 z)^{3}}+\ldots\right)
$$

where $\mu=4 \nu^{2}$. Using (18) and (29), we obtain the following representation (see Donoghue [11], p. 295)

$$
f_{\alpha}(|\lambda|)=c_{1}(\alpha)|\lambda|^{\alpha-1}(1-\theta(|\lambda|)), 0<\alpha<1, \lambda \in \mathbb{R}
$$

where $\theta(|\lambda|) \rightarrow 0$ as $|\lambda| \rightarrow 0$. The spectral density $f_{\alpha}(|\lambda|), \lambda \in \mathbb{R}$, corresponding to the covariance function (17) is the Bessel potential of order $\alpha \in(0,1)$ (see for example, Donoghue [11], p. 294), that is,

$$
f_{\alpha}(\rho)=\frac{1}{(4 \pi)^{1 / 2} \Gamma\left(\frac{\alpha}{2}\right)} \int_{0}^{\infty} e^{-\rho^{2} /(4 \mu)} e^{-\mu} \mu^{(\alpha-1) / 2} \frac{d \mu}{\mu}, \rho=|\lambda| .
$$

Therefore, for the spectral density $f_{\alpha}(|\lambda|), \lambda \in \mathbb{R}$, the following convolution equation holds:

$$
f_{\alpha+\beta}(\lambda)=\int_{-\infty}^{\infty} f_{\alpha}\left(\lambda^{\prime}\right) f_{\beta}\left(\lambda^{\prime}-\lambda\right) d \lambda^{\prime}, \alpha>0, \beta>0
$$

From (2) we obtain

$$
B^{m}(t)=\int_{-\infty}^{\infty} \cos (\lambda t) f^{* m}(\lambda) d \lambda
$$

where the convolutions $f^{* m}(\lambda)$ are defined recursively as

$$
f^{* 1}(\lambda)=f(\lambda), f^{* m}(\lambda)=\int f^{*(m-1)}\left(\lambda^{\prime}\right) f\left(\lambda^{\prime}-\lambda\right) d \lambda^{\prime}, m=2,3, \ldots
$$

In particular, we obtain from (22) the following elegant formula for the spectral density (18):

$$
f^{* m}(\lambda)=f_{m \alpha}(\lambda), \lambda \in \mathbb{R}, 0<\alpha m<1,
$$

where $f_{m \alpha}(\lambda), \lambda \in \mathbb{R}$ is given by (18). Using (18)-(21) and the relation

$$
\int_{0}^{\infty} \lambda^{-2 \gamma} \sin ^{2} \lambda d \lambda=-4 \sin (\gamma \pi) \Gamma(1-2 \gamma) 2^{\gamma-2}, \gamma \in\left(\frac{1}{2}, \frac{3}{2}\right)
$$

we obtain from Lemma 4.2 (see Section 4 below) with $U(z)=\sin ^{2} z$ and $\delta=m \alpha-3$, 
$0<\alpha m<1$ the following formula:

as $T \rightarrow \infty$.

$$
\begin{gathered}
\operatorname{var}\left[\int_{0}^{T} H_{m}(\xi(t)) d t\right]=2 m ! \int_{0}^{\infty} \sin ^{2}\left(\frac{\lambda T}{2}\right)\left(\frac{\lambda}{2}\right)^{-2} f_{m \alpha}(\lambda) d \lambda \\
=T^{2-m \alpha} m ! c_{2}(m, \alpha)(1+o(1))
\end{gathered}
$$

Let $X$ and $Y$ be arbitrary random variables. Introduce a uniform (or Kolmogorov's) distance between the distributions of the random variables $X$ and $Y$ via the formula

$$
\mathscr{G}(X, Y)=\sup _{z}|P(X \leq z)-P(Y \leq z)| .
$$

The main result of this paper describes the rate of convergence (as $T \rightarrow \infty)$ in Theorem 2.1 with $m=2$ and is contained in the following.

Theorem 3.1: Let assumptions $A, B, B^{\prime}$ and $C$ hold with $m=2$ and $\alpha \in(0,1 / 3)$. Then

$$
\lim _{T \rightarrow \infty} \sup T^{\alpha / 3} \mathcal{S O}_{0}\left(\frac{1}{T^{1-\alpha}} \int_{0}^{T}\left[G(\xi(t))-C_{0}\right] d t, R_{2}(1)\right)
$$

exists and does not exceed the constant

$$
\left\{\frac{2}{(1-3 \alpha)(2-3 \alpha)}\left[\int_{-\infty}^{\infty} G^{2}(u) \varphi(u) d u-C_{0}^{2}-\frac{C_{2}^{2}}{2}\right]\right\}^{1 / 3}\left(\frac{3}{2}\right)^{2 / 3} \frac{C_{2}^{2 / 3}\left[c_{1}(\alpha)\right]^{2 / 3}}{\left(\nu_{p} \nu_{q}\right)^{1 / 3}}
$$

where $C_{0}, C_{2}$ are defined in (6) and the constant $c_{1}(\alpha)$ is defined in (4). The numbers $\nu_{p}$ and $\nu_{q}$ are defined in (16), and the random variable $R_{2}(1)$ which has the Rosenblatt distribution is defined in (10) or (12) and (13).

Remark 3.3: Our methodology in principle, is applicable to more general Gaussian processes in continuous time. For this, we have to replace condition $\mathrm{C}$ by a more general condition which can be given in the spectral form, such as (21), together with the type of results of Lemma 4.5 and a precise behavior of the spectral density near infinity (for example, $f(\lambda)=O\left(|\lambda|^{-1-\alpha}\right.$ ) as $\left.|\lambda| \rightarrow \infty\right)$. Then, instead of the convolution property (22), we may use the Riesz Composition Formula (see Lemma 4.6 below) for an investigation of the asymptotic behavior of convolutions of spectral density. Lemma 4.2 can next be used again to obtain the asymptotic formulae similar to (9) but in terms of the spectral density. Then the proof can be completed by following the same principal steps. We will address this approach in a separate paper together with a generalization to random fields.

\section{Proof of the Main Result}

Before proving Theorem 3.1, we state some well-known results.

Lemma 4.1: Let $(\xi, \eta)$ be a Gaussian vector with $E \xi=E \eta=0, E \xi^{2}=E \eta^{2}=1$, $E \xi \eta=\rho$. Then for all $m \geq 0, q \geq 0$,

$$
E H_{m}(\xi) H_{q}(\eta)=\delta_{m}^{q} \rho^{m} m !
$$


where $\delta_{m}^{q}$ is the Kronecker symbol.

The proof of Lemma 4.1 is well-known (see for example, Ivanov and Leonenko [17], p. 55).

Lemma 4.2: Consider the integral

Suppose that

$$
S(T)=\int_{0}^{\infty} U(\lambda T) \lambda^{\delta} \psi(\lambda) d \lambda .
$$

(a) the function $\psi(\lambda)$ is continuous in a neighborhood of zero, $\psi(0) \neq 0$ and

Then $\psi(\lambda)$ is bounded on $[0, \infty)$;

$$
\int_{0}^{\infty} U(z) z^{\delta} d z=\gamma(\delta), \int_{0}^{\infty}|U(z)| z^{\delta} d z<\infty
$$

$$
S(T)=T^{-\delta-1} \psi(0) \gamma(\delta)(1+o(1)) \text { as } T \rightarrow \infty .
$$

The proof of Lemma 4.2 can be found, for example, in Ivanov and Leonenko [17], pp. 29-30.

The following lemma is due to Petrov [28], p. 29.

Lemma 4.3: Let $X, Y, Z$ be arbitrary random variables such that

$$
|P(X \leq z)-P(Z \leq z)| \leq K
$$

where $K$ is a constant. Then for any $\varepsilon>0$

where

$$
\mathscr{K}(X+Y, Z) \leq K+L_{\varepsilon}+P(|Y| \geq \varepsilon)
$$

and

$$
L_{\varepsilon}=\max \sup _{z}\{|T(z+\varepsilon)-T(z)|,|T(z-\varepsilon)-T(z)|\}
$$

$$
T(z)=P(Z \leq z) \text {. }
$$

We now formulate the following statement.

Lemma 4.4: Consider the random variable $R_{2}(1)$ which has the Rosenblatt distribution (see (10)). Then there exists a density function

$$
p(z)=\frac{d}{d z} P\left(R_{2}(1) \leq z\right) \leq c_{3}=\frac{C_{2} c_{1}(\alpha)}{2 \sqrt{\nu_{p} \nu_{q}}},
$$

where $C_{2}$ is defined in (6), the constant $c_{1}(\alpha)$ is defined in (4) and $\nu_{p}$ and $\nu_{q}$ are defined in (16).

Proof: Using representations (13), we obtain that

where

$$
\overline{R_{2}}=\eta_{1}+\eta_{2}
$$

$$
\eta_{1}=\nu_{p}\left(X_{p}^{2}-1\right)+\nu_{q}\left(X_{q}^{2}-1\right)=\nu_{q}\left(\beta X_{p}^{2}+X_{q}^{2}\right)-\left(\nu_{p}+\nu_{q}\right)
$$

and

$$
\eta_{2}=\sum_{k \neq k \neq q} \nu_{k}\left(X_{k}^{2}-1\right)
$$


The random variables $X_{p}$ and $X_{q}$ are independent standard normal. Thus the density function of the random variable $\zeta=\beta X_{p}^{2}+X_{q}^{2}$ is of the form

$$
\begin{gathered}
p_{\zeta}(u)=\int_{0}^{u} p_{X_{p}^{2}}(u-x) p_{\beta X_{p}^{2}}(x) d x \\
=\frac{1}{2 \Gamma^{2}(1 / 2) \beta^{1 / 2}} e^{-u / 2} \int_{0}^{u}(u-x)^{-1 / 2} x^{-1 / 2} e^{-(1 / \beta-1) x / 2} d x \\
\leq \frac{1}{2\left(\nu_{p} / \nu_{q}\right)^{1 / 2}} .
\end{gathered}
$$

Thus, the density function of the random variable $\eta_{1}$ is also bounded:

$$
p_{\eta_{1}}(u) \leq \frac{1}{2 \sqrt{\nu_{p} \nu_{q}}} \text {. }
$$

From (24) and (25) we obtain that there exists a density function of Rosenblatt process $\overline{R_{2}}$ :

$$
p_{\overline{R_{s}}}(x)=\frac{d}{d x} P\left(\overline{R_{2}} \leq x\right)=\int p_{\eta_{1}}(x-y) d F_{\eta_{2}}(y) \leq \frac{1}{2 \sqrt{\nu_{p} \nu_{q}}}
$$

where $F_{\eta_{2}}(y)=P\left(\eta_{2} \leq y\right)$ and $p_{\eta_{1}}(x)$ is the density function of the random variable $\eta_{1}$. The density function of the random variable $R_{2}(1)$ defined in (10), also exists and is bounded by a constant $c_{3}$, defined in (23).

Some further information on the density function of the Rosenblatt distribution can be found in Albin [1].

Lemma 4.5: Let $f_{\alpha}(\lambda), \lambda \in \mathbb{R}$, be a spectral density given by (18). Then the asymptotic relation (21) holds as $|\lambda| \rightarrow 0$ with

$$
\theta(|\lambda|)=K|\lambda|^{1-\alpha}(1+o(1))
$$

where $K$ is a positive constant.

Proof: From Formula 4 of 3.773 of Gradshteyn and Ryzhik [15], we obtain

$$
\begin{gathered}
\int_{0}^{\infty} \frac{\cos \lambda t}{\left(1+t^{2}\right)^{\alpha / 2}} d t=\frac{1}{2} B\left(\frac{1}{2}, \frac{\alpha-1}{2}\right)_{1} F_{2}\left(\frac{1}{2} ; \frac{3-\alpha}{2}, \frac{1}{2} ; \frac{\lambda^{2}}{4}\right) \\
+\sqrt{\pi} \frac{\lambda^{\alpha-1}}{4^{\alpha / 2}} \frac{\Gamma\left(\frac{1-\alpha}{2}\right)}{\Gamma\left(\frac{\alpha}{2}\right)}{ }_{1} F_{2}\left(\frac{\alpha}{2} ; \frac{\alpha}{2}, \frac{\alpha+1}{2} ; \frac{\lambda^{2}}{4}\right), \alpha>0, \\
=\frac{1}{2} B\left(\frac{1}{2}, \frac{\alpha-1}{2}\right)\left(1+\frac{1 / 2}{\frac{3-\alpha}{2} \cdot \frac{1}{2}} \frac{\lambda^{2}}{4}+\ldots\right)+\sqrt{\pi} \frac{\lambda^{\alpha-1}}{4^{\alpha / 2}} \frac{\Gamma\left(\frac{1-\alpha}{2}\right)}{\Gamma\left(\frac{\alpha}{2}\right)}\left(1+\frac{\alpha / 2}{\frac{\alpha}{2} \cdot \frac{\alpha+1}{2}} \frac{\lambda^{2}}{4}+\ldots\right),
\end{gathered}
$$

$\alpha>0$, where ${ }_{1} F_{2}$ is a hypergeometric function. The statement of Lemma 4.5 now follows by direct computations.

The following statement is known as the Riesz Composition Formula (see, for example, Plessis [29], pp. 71-72).

Lemma 4.6: For $0<\alpha<1,0<\beta<1,0<\alpha+\beta<1$, the following identity holds: 


$$
c_{4}(\alpha, \beta)|x-y|^{\alpha+\beta-1}=\int_{-\infty}^{\infty}|x-z|^{\alpha-1}|z-y|^{\beta-1} d z
$$

where

$$
c_{4}(\alpha, \beta)=\frac{\left.\sqrt{\pi} \Gamma\left(\frac{\alpha}{2}\right) \Gamma\left(\frac{\beta}{2}\right) \Gamma \frac{1-\alpha-\beta}{2}\right)}{\Gamma\left(\frac{1-\alpha}{2}\right) \Gamma\left(\frac{1-\beta}{2}\right) \Gamma\left(\frac{\alpha+\beta}{2}\right)} .
$$

Proof of Theorem 3.1: Let $L_{2}(\Omega)$ be a Hilbert space of random variables with finite second moments. From (6) and (7), we obtain the following expansion in $L_{2}(\Omega)$ :

$$
\left.\left.\int_{0}^{T}\right] G(\xi(t))-C_{0}\right] d t=\sum_{k=m}^{\infty} \frac{C_{m}}{m !} \zeta_{m}(T)
$$

where

$$
\zeta_{k}(T)=\int_{0}^{T} H_{k}(\xi(t)) d t
$$

and, by Lemma 4.1,

$$
E \zeta_{k}(T) \zeta_{r}(T)=k ! \delta_{k}^{r} \sigma_{k}^{2}(T), k \geq m
$$

where by (17)

$$
\begin{aligned}
\sigma_{k}^{2}(T)= & E\left[\int_{0}^{T} H_{k}(\xi(t)) d t\right]^{2}=\int_{0}^{T} \int_{0}^{T} B^{k}(|t-s|) d t d s \\
& =T^{2-k \alpha} \int_{0}^{1} \int_{0}^{1} B^{k}(T|t-s|) \cdot T^{k \alpha} d t d s \\
& =T^{2-k \alpha} c_{2}(k, \alpha)(1+o(1)) \text { as } T \rightarrow \infty
\end{aligned}
$$

$c_{2}(k, \alpha)$ being defined in $(9)$ and $0<k \alpha<1$.

In order to apply Lemma 4.3 with $Z=R_{2}(1)$, we represent

where

$$
Y_{T}(1)=\frac{1}{T^{1-\alpha}} \int_{0}^{T}\left[G(\xi(t))-C_{0}\right] d t=X_{T}+Y_{T}
$$

and

$$
X_{T}=\frac{C_{2}}{2} \int_{0}^{T} H_{2}(\xi(t)) d t / T^{1-\alpha}
$$

$$
Y_{T}=\left[\sum_{k=3}^{\infty} \frac{C_{k}}{k !} \zeta_{k}(T)\right] / T^{1-\alpha}
$$

By (17), Lemma 4.1 and the Parseval identity, we obtain for $0<\alpha<1 / 3$ that

$$
\operatorname{var} Y_{T}=\sum_{k=3}^{\infty} \frac{C_{k}^{2}}{k !} \sigma_{k}^{2}(T) / T^{2-2 \alpha}
$$




$$
\begin{aligned}
\leq\left(\sigma_{3}^{2}(T) / T^{2-2 \alpha}\right) c(G) & \leq \frac{c(G)}{T^{\alpha}} \int_{0}^{1} \int_{0}^{1}\left[T^{\alpha} B(T|t-s|)\right]^{3} d t d s \\
& =\frac{c(G)}{T^{\alpha}} g_{T}(\alpha) .
\end{aligned}
$$

We have

$$
\lim _{T \rightarrow \infty} g_{T}(\alpha)=c_{2}(3, \alpha)
$$

the constant $c_{2}(3, \alpha)$ being defined in $(9)$, and the constant

$$
c(G)=\sum_{k=3}^{\infty} C_{k}^{2} / k !=\int_{-\infty}^{\infty} G^{2}(u) \varphi(u) d u-C_{0}^{2}-\frac{C_{2}^{2}}{2}
$$

Using Lemma 4.4 we have the following estimate for $L_{\varepsilon}$ defined in Lemma 4.3:

$$
L_{\varepsilon} \leq \varepsilon c_{3}
$$

where $c_{3}$ is given in (23).

By Lemma 4.3 (with $X=X_{T}, \quad Y=Y_{T}, \quad Z=R_{2}(1)$ ) and the Chebyshev inequality, we obtain from (27)-(30), that

$$
\mathscr{K}\left(X_{T}+Y_{T}, R_{2}(1)\right) \leq \mathfrak{K}\left(X_{T}, R_{2}(1)\right)+c_{3} \varepsilon+\frac{1}{\varepsilon^{2}} \frac{c(G)}{T^{\alpha}} g_{T}(\alpha) .
$$

Using (5), condition C and Itô's formula (see for example, Taqqu [37], Major [25]), we obtain

$$
H_{2}(\xi(t))=\int_{\mathbb{R}^{2}}^{\prime} e^{i\left(\lambda_{1}+\lambda_{2}\right) t} \sqrt{f_{\alpha}\left(\lambda_{1}\right) f_{\alpha}\left(\lambda_{2}\right)} W\left(d \lambda_{1}\right) W\left(d \lambda_{2}\right),
$$

where $f_{\alpha}(\lambda)$ is defined in (18).

Using the self-similarity property of the Gaussian white noise (formally, $W(a d \lambda) \stackrel{d}{=} \sqrt{a} W(d \lambda)$, where $\stackrel{d}{=}$ stands for equality of distributions), we obtain from (7) and (32) the following representation:

$$
\begin{gathered}
X_{T} \stackrel{d}{=} \frac{C_{2}}{2} \int_{\mathbb{R}^{2}}^{\prime} \frac{e^{i\left(\lambda_{1}+\lambda_{2}\right)}-1}{i\left(\lambda_{1}+\lambda_{2}\right)} \frac{1}{\left|\lambda_{1} \lambda_{2}\right|^{(1-\alpha) / 2}} \\
\times\left(\left|\frac{\lambda_{1}}{T}\right|\left|\frac{\lambda_{2}}{T}\right|\right)^{\frac{1-\alpha}{2}} \sqrt{f_{\alpha}\left(\frac{\lambda_{1}}{T}\right) f_{\alpha}\left(\frac{\lambda_{2}}{T}\right) W\left(d \lambda_{1}\right) W\left(d \lambda_{2}\right) .}
\end{gathered}
$$

From (10) and (33), we have

$$
\begin{gathered}
X_{T}^{\prime}=X_{T}-R_{2}(1)=\frac{C_{2}}{2} \int_{\mathbb{R}^{2}}^{\prime} \frac{e^{i\left(\lambda_{1}+\lambda_{2}\right)}-1}{i\left(\lambda_{1}+\lambda_{2}\right)} \frac{1}{\left|\lambda_{1} \lambda_{2}\right|^{(1-\alpha) / 2}} \\
\times\left(\left(\left|\frac{\lambda_{1}}{T}\right|\left|\frac{\lambda_{2}}{T}\right|\right)^{\frac{1-\alpha}{2}} \sqrt{f_{\alpha}\left(\frac{\lambda_{1}}{T}\right) f_{\alpha}\left(\frac{\lambda_{2}}{T}\right)}-c_{1}(\alpha)\right) W\left(d \lambda_{1}\right) W\left(d \lambda_{2}\right),
\end{gathered}
$$


and by the properties of multiple stochastic integrals (see, for example, Major [25]), we obtain

where

$$
\operatorname{var} X_{T}^{\prime}=\frac{1}{T^{\alpha}} \frac{C_{2}^{2}}{4} \int_{\mathbb{R}^{2}}\left|\frac{e^{i\left(\lambda_{1}+\lambda_{2}\right)}-1}{i\left(\lambda_{1}+\lambda_{2}\right)}\right|^{2} \frac{Q_{T}\left(\lambda_{1}, \lambda_{2}\right)}{\left|\lambda_{1} \lambda_{2}\right|^{1-\alpha}} d \lambda_{1} d \lambda_{2}
$$

$$
Q_{T}\left(\lambda_{1}, \lambda_{2}\right)=T^{\alpha}\left[\left(\left|\frac{\lambda_{1}}{T}\right|\left|\frac{\lambda_{2}}{T}\right|\right)^{\frac{1-\alpha}{2}} \sqrt{f_{\alpha}\left(\frac{\lambda_{1}}{T}\right) f_{\alpha}\left(\frac{\lambda_{2}}{T}\right)}-c_{1}(\alpha)\right]^{2} .
$$

From (18)-(21), we can see that the function

$$
T^{-\alpha} Q_{T}\left(\lambda_{1}, \lambda_{2}\right) \leq K_{1}
$$

Consider now

$$
\begin{aligned}
& \int_{\mathbb{R}^{2}}\left|\frac{e^{i\left(\lambda_{1}+\lambda_{2}\right)}-1}{i\left(\lambda_{1}+\lambda_{2}\right)}\right|^{2} \frac{Q_{T}\left(\lambda_{1}, \lambda_{2}\right)}{\left|\lambda_{1} \lambda_{2}\right|^{1-\alpha}} d \lambda_{1} d \lambda_{2}=\iint_{\left|\lambda_{1}\right| \leq T^{1-\alpha},\left|\lambda_{2}\right| \leq T^{1-\alpha}}^{\cdots} \ldots \\
& +\iint \\
& \ldots+2 \iint \\
& \left|\lambda_{1}\right|>T^{1-\alpha},\left|\lambda_{2}\right|>T^{1-\alpha} \quad\left|\lambda_{1}\right|>T^{1-\alpha},\left|\lambda_{2}\right| \leq t^{1-\alpha} \\
& =I_{1}+I_{2}+I_{3} \text {. }
\end{aligned}
$$

By Lemma 4.5 , for $\left|\frac{\lambda_{i}}{T}\right| \leq \frac{1}{T^{\alpha}}, i=1,2$ and $T \rightarrow \infty$, we obtain

$$
\begin{aligned}
& Q_{T}\left(\lambda_{1}, \lambda_{2}\right)=T^{\alpha} c_{1}(\alpha)\left[\sqrt{\left(1-\theta\left(\left|\frac{\lambda_{1}}{T}\right|\right)\right)\left(1-\theta\left(\left|\frac{\lambda_{2}}{T}\right|\right)\right)}-1\right]^{2} \\
= & T^{\alpha} c_{1}(\alpha)\left[-\frac{1}{2}\left|\frac{\lambda_{1}}{T}\right|^{1-\alpha}-\frac{1}{2}\left|\frac{\lambda_{2}}{T}\right|^{1-\alpha}+\frac{1}{4}\left(\left|\frac{\lambda_{1}}{T}\right|\left|\frac{\lambda_{2}}{T}\right|\right)^{1-\alpha}+o(1)\right]^{2} \\
= & T^{\alpha} c_{1}(\alpha)\left[\frac{1}{4}\left|\frac{\lambda_{1}}{T}\right|^{2(1-\alpha)}+\frac{1}{4}\left|\frac{\lambda_{2}}{T}\right|^{2(1-\alpha)}+\frac{1}{4}\left(\left|\frac{\lambda_{1}}{T}\right|\left|\frac{\lambda_{2}}{T}\right|\right)^{1-\alpha}+o(1)\right]
\end{aligned}
$$

using the approximation $(1-x)^{1 / 2}=1-\frac{1}{2} x+o(x)$. Note that, for $\left|\frac{\lambda_{i}}{T}\right| \leq \frac{1}{T^{\alpha}}$, $i=1,2$,

$$
T^{\alpha}\left[\left|\frac{\lambda_{1}}{T}\right|^{2(1-\alpha)}+\left|\frac{\lambda_{2}}{T}\right|^{2(1-\alpha)}+\left(\left|\frac{\lambda_{1}}{T}\right|\left|\frac{\lambda_{2}}{T}\right|\right)^{1-\alpha}\right] \leq K_{2} T^{-\alpha(1-2 \alpha)}
$$

Thus,

$$
I_{1}=K_{3} T^{-\alpha(1-2 \alpha)}(1+o(1)) \text { as } T \rightarrow \infty \text { and for } 0<\alpha<\frac{1}{2}
$$


Using Lemma 4.6 and change of variables: $\lambda_{1}+\lambda_{2}=u, \lambda_{2}=\nu$, we obtain for $I_{2}$ the following estimate:

$$
\begin{gathered}
I_{2} \leq K_{1} T^{\alpha} \int_{\left|\lambda_{1}\right|>T^{1-\alpha},\left|\lambda_{2}\right|>T^{1-\alpha}} \frac{\sin ^{2} \frac{\lambda_{1}+\lambda_{2}}{2}}{\left(\frac{\lambda_{1}+\lambda_{2}}{2}\right)^{2}} \frac{d \lambda_{1} d \lambda_{2}}{\left|\lambda_{1} \lambda_{2}\right|^{1-\alpha}} \\
\leq K_{4} T^{\alpha} \int_{2 T^{1-\alpha}}^{\infty} \frac{d \nu}{\nu^{1-\alpha}} \int_{T^{1-\alpha}}^{\infty} \frac{1}{T^{1+\alpha}} \frac{d u}{|u|^{1-\alpha}|u-\nu|^{1-\alpha}} \\
\leq K_{5} T^{-1}, \quad 0<\alpha<\frac{1}{3}
\end{gathered}
$$

Combining the arguments for estimates of $I_{1}$ and $I_{2}$, we obtain

$$
I_{3}=K_{6} T^{-\alpha(1-2 \alpha)}(1+o(1)) \text { as } T \rightarrow \infty .
$$

From (35)-(38), we have for $0<\alpha<1 / 3$,

$$
\mu_{T}(\alpha)=\frac{C_{2}^{2}}{4} \int_{\mathbb{R}^{2}}\left|\frac{e^{i\left(\lambda_{1}+\lambda_{2}\right)}-1}{i\left(\lambda_{1}+\lambda_{2}\right)}\right|^{2} \frac{Q_{T}\left(\lambda_{1}, \lambda_{2}\right)}{\left|\lambda_{1} \lambda_{2}\right|^{1-\alpha}} d \lambda_{1} d \lambda_{2} \rightarrow 0
$$

as $T \rightarrow \infty$.

We are now in a position to apply Lemma 4.3 again with $X=R_{2}(1), Y=X_{T}^{\prime}$ and $Z=R_{2}(1)$. In this case, we can choose $K=0$ in the statement of Lemma 4.3. Thus, for any $\varepsilon>0$,

$$
\begin{gathered}
\mathscr{K}\left(X_{T}, R_{2}(1)\right) \leq c_{3} \varepsilon+P\left\{\left|X_{T}^{\prime}\right| \geq \varepsilon\right\} \\
\leq c_{3} \varepsilon+\frac{1}{\varepsilon^{2}} \operatorname{var} X_{T}^{\prime}
\end{gathered}
$$

where $c_{3}$ is defined in (23) and $\operatorname{var} X_{T}^{\prime}$ is given by (34).

From (31), (34) and (39) we obtain, for any $\varepsilon>0$,

$$
\mathscr{K}_{0}\left(X_{T}+Y_{T}, R_{2}(1)\right) \leq 2 c_{3} \varepsilon+\frac{1}{\varepsilon^{2}} \frac{1}{T^{\alpha}}\left(c(G) g_{T}(\alpha)+\mu_{T}(\alpha)\right),
$$

where $\mu_{T}(\alpha)$ is defined in (38) and $g_{T}(\alpha)$ is defined in (27) and (28).

In order to minimize the right-hand side of $(40)$, we set

$$
\varepsilon=\left\{2 T^{-\alpha}\left(c(G) g_{T}(\alpha)+\mu_{T}(\alpha)\right) / 2 c_{3}\right\}^{1 / 3} .
$$

We then obtain the following inequality:

$$
\mathscr{K}\left(\frac{1}{T^{1-\alpha}} \int_{0}^{T}\left[G(\xi(t))-C_{0}\right] d t, R_{2}(1)\right) \leq \frac{1}{T^{\alpha / 3}}\left[c(G) g_{T}(\alpha)+\mu_{T}(\alpha)\right]^{1 / 3}\left(3 c_{3}^{2 / 3}\right)
$$

where $g_{T}(\alpha) \rightarrow c_{2}(3, \alpha)$ and $\mu_{T}(\alpha) \rightarrow 0$ as $T \rightarrow \infty$. Theorem 3.1 now follows directly from the above inequality. 
Remark 4.1: It should be noted that the Rosenblatt distribution is absolutely continuous (see (13), (23) or Albin [1]). Hence convergence in distribution to the Rosenblatt distribution implies convergence of the Kolmogorov distance to zero.

\section{Extension}

Theorem 3.1 gives the convergence rate to zero of the Kolmogorov distance between normalized functionals of random processes with LRD and the Rosenblatt distribution only for $\alpha \in(0,1 / 3)$. On the other hand, it follows from Theorem 2.1 that the convergence of the Kolmogorov distance to zero holds for $\alpha \in(0,1 / 2)$. As it turns out, our method is also applicable for the interval $\alpha \in[1 / 3,1 / 2)$, but the outcome is a slower convergence rate.

Theorem 5.1: Let assumptions $A, B, B^{\prime}$ and $C$ hold with $m=2$ and $\alpha \in\left[\frac{1}{3}, \frac{1}{2}\right)$. Then

$$
\limsup _{T \rightarrow \infty} T^{\frac{\alpha(1-2 \alpha)}{3(1+\alpha)}} \mathscr{G}\left(\frac{1}{T^{1-\alpha}} \int_{0}^{T}\left[G(\xi(t))-C_{0}\right] d t, R_{2}(1)\right)
$$

exists and does not exceed the constant

$$
\left\{2+\frac{1}{2(1-2 \alpha)(1-\alpha)}\left[\int_{-\infty}^{\infty} G^{2}(u) \varphi(u) d u-C_{0}^{2}-\frac{C_{2}^{2}}{2}\right]\right\}^{\frac{1}{3}}\left(\frac{3}{2}\right)^{\frac{2}{3}} C_{2}^{\frac{2}{3}}\left[c_{1}(\alpha)\right]^{\frac{2}{3}} /\left(\nu_{p} \nu_{q}\right)^{\frac{1}{3}}
$$

Proof: We follow the scheme of the proof of Theorem 3.1, incorporating necessary modifications. In particular, we represent

where

$$
Y_{T}(1)=X_{T}+Y_{T}
$$

$$
\operatorname{var} Y_{T} \leq \frac{c(G)}{T^{2-2 \alpha}} \sigma_{3}^{2}(T)
$$

Observe that

$$
\delta=\frac{1-2 \alpha}{1+\alpha} \in(0,1), \delta<1-2 \alpha, \alpha \delta \leq 1-2 \alpha-\delta
$$

for $\alpha \in\left[\frac{1}{3}, \frac{1}{2}\right)$. Thus,

$$
\begin{aligned}
\sigma_{3}^{2}(T)= & \int_{0}^{T} \int_{0}^{T} B^{3}(|t-s|) d t d s=2 T \int_{0}^{T}\left(1-\frac{\tau}{T}\right) B^{3}(\tau) d \tau \\
& =2 T\left[\int_{0}^{T^{\delta}}+\int_{T^{\delta}}^{T}\right]\left(1-\frac{\tau}{T}\right) B^{3}(\tau) d \tau \\
& \leq 2 T^{1+\delta}+B\left(T^{\delta}\right) 2 T \int_{T^{\delta}}^{T} B^{2}(\tau)\left(1-\frac{\tau}{T}\right) d t
\end{aligned}
$$




$$
\leq 2 T^{1+\delta}+B\left(T^{\alpha}\right) T^{2-2 \alpha} \int_{0}^{1} \int_{0}^{1}\left(B(T|t-s|) T^{2}\right)^{\alpha} d t d s
$$

and using (41), we have

$$
\operatorname{var} Y_{T} \leq \frac{2 c(G)}{T^{1-2 \alpha-\delta}}+\frac{c(G)}{T^{\alpha \delta}} \tilde{g}_{T}(\alpha)
$$

where as $T \rightarrow \infty$

$$
\begin{gathered}
\tilde{g}_{T}(\alpha)=T^{\alpha \delta} B\left(T^{\delta}\right) \int_{0}^{1} \int_{0}^{1}\left[B(T|t-s|) T^{\alpha}\right]^{2} d t d s \\
\rightarrow c_{2}(2, \alpha)
\end{gathered}
$$

By Lemma 4.3 we obtain in a similar way to (40) that

$$
\mathscr{K}\left(X_{T}+Y_{T}, R_{2}(1)\right) \leq \mathscr{K}\left(X_{T}(1), R_{2}(1)\right)+c_{3} \varepsilon+\frac{1}{\varepsilon^{2}} \frac{c(G)}{T^{\alpha \delta}}\left[2+\tilde{g}_{T}(\alpha)\right]
$$

Following the scheme of the proof of Theorem 3.1, we obtain the estimate (34) in which $\frac{1}{T^{\alpha}}$ is replaced by $\frac{1}{T^{\alpha \delta}}$. The estimate (35) holds for $\alpha \in\left(0, \frac{1}{2}\right)$. Using the following formula (see Gradshteyn and Ryzhik [15], formula 2 of 3.194)

$$
\int_{u}^{\infty} \frac{x^{\mu-1}}{(1+\beta x)^{\nu}} d x=\frac{u^{\mu-\nu}}{\beta^{\nu}(\nu-\mu)}{ }_{2} F_{1}\left(\nu, \nu-\mu ; \nu-\mu+1 ;-\frac{1}{\beta u}\right)
$$

with $\operatorname{Re} \nu>\operatorname{Re} \mu$, we obtain for $\alpha \in\left(0, \frac{1}{2}\right)$

$$
\int_{\left|\lambda_{1}\right|>T^{1-\alpha}} \frac{\sin ^{2} \frac{\lambda_{1}+\lambda_{2}}{2}}{\left(\frac{\lambda_{1}+\lambda_{2}}{2}\right)^{2}} \frac{d \lambda_{1}}{\left|\lambda_{1}\right|^{1-\alpha}} \leq K_{7} T^{\alpha-2}{ }_{2} F_{1}\left(2,2-\alpha ; 3-\alpha ;-\frac{\lambda_{2}}{T^{1-\alpha}}\right) .
$$

Therefore,

$$
I_{2} \leq K_{8} T^{\alpha} \int_{2 T^{1-\alpha}}^{\infty} \frac{d \lambda_{2}}{\lambda_{2}^{1-\alpha}} T^{\alpha-2}{ }_{2} F_{1}\left(2,2-\alpha, 3-\alpha,-\frac{\lambda_{2}}{T^{1-\alpha}}\right)
$$

and for $\alpha \in\left(0, \frac{1}{2}\right)$

$$
\lim _{T \rightarrow \infty} I_{2}=0
$$

Similarly,

$$
\lim _{T \rightarrow \infty} I_{3}=0 \text { for } \alpha \in\left(0, \frac{1}{2}\right)
$$

Thus from (43)

$$
\mathscr{G}\left(X_{T}, R_{2}(1)\right) \leq 2 c_{3} \varepsilon+\frac{1}{\varepsilon^{2}} \frac{1}{T^{\alpha \delta}}\left(\left(c(G)\left(2+\tilde{g}_{T}(\alpha)\right)\right)+\mu_{T}(\alpha)\right),
$$


where

$$
\lim _{T \rightarrow \infty} \mu_{T}(\alpha)=0
$$

Let us set

$$
\varepsilon=\left(2 T-\alpha \delta \frac{c(G)\left(2+\tilde{g}_{T}(\alpha)\right)+\mu_{T}(\alpha)}{2 c_{3}}\right)^{\frac{1}{3}} .
$$

We then obtain the following inequality:

$$
\begin{aligned}
& \mathscr{G}\left(\frac{1}{T^{1-\alpha}} \int_{0}^{T}\left[G(\xi(t))-C_{0}\right] d t, R_{2}(1)\right) \\
& \left.\leq \frac{1}{T^{\frac{\alpha \delta}{3}}} c(G)\left(2+\tilde{g}_{T}(\alpha)\right)+\mu_{t}(\alpha)\right]^{\frac{1}{3}}\left(2 c_{3}^{\frac{2}{3}}\right),
\end{aligned}
$$

with

$$
\delta=\frac{1-2 \alpha}{1+\alpha}
$$

From (42)-(45), Theorem 5.1 follows.

\section{Concluding Remarks}

This paper addresses the issue of measuring the speed of convergence to the Rosenblatt distribution, as measured by the Kolmogorov distance, for some functionals of nonlinear transformations of long-range dependent Gaussian processes with Hermite rank $m=2$. Our method is based on a direct probabilistic analysis of the main term $(m=2)$ as well as the second term $(m=3)$. Due to the nature of limiting laws in the situation of LRD, it is not straightforward to present an argument on the sharpness of the results as in the traditional situation of short-range dependence. In particular, the rate of convergence in Theorem 5.1 is not optimal, hence yields a gap in the rate of convergence at $\alpha=1 / 3$ between Theorems 3.1 and 5.1. However, the paper takes the first step towards solving the important and difficult problem of sharp convergence rate in non-central limit theorems.

The method of this paper in fact is general. It can be applied to nonlinear functionals of non-Gaussian random processes with LRD and special bilinear expansions of their bivariate densities in orthogonal polynomials such as ChebyshevHermite polynomials, Laguerre polynomials. In particular, the rate of convergence to the non-Gaussian Laguerre processes with Laguerre rank $m=1$ has been obtained in Anh and Leonenko [4] (see also Leonenko [20]).

\section{Acknowledgements}

We would like to tank M. Taqqu, M. Benšsic and I. Ostrovskii for useful discussions on the topic. The authors are also grateful to the referees for their careful reading of the manuscript and suggestions for improvement. 


\section{References}

[1] Albin, J.M.P., A note on Rosenblatt distributions, Stats. and Prob. Letters 40 (1998), 83-91.

[2] Anh, V.V. and Heyde, C.C. (editors), Special Issue on Long-Range Dependence, J. Statist. Planning \& Inference 801999.

[3] Anh, V.V., Angulo, J.M. and Ruiz-Medina, M.D., Possible long-range dependence in fractional random fields, J. Statist. Planning $\mathscr{E}$ Inference 80 (1999), 95-110.

[4] Anh, V.V. and Leonenko, N.N., Non-Gaussian scenarios for the heat equation with singular initial conditions, Stoch. Proc. Appl. 84 (1999), 91-114.

[5] Barndorff-Nielsen, O.E., Processes of normal inverse Gaussian type, Finance and Stoch. 2 (1998), 41-68.

[6] Beran, J., Statistics for Long-Memory Processes, Chapman and Hall, New York 1994.

[7] Berman, S.M., High level sojourns for strongly dependent Gaussian processes, Z. Wahrsch. Verw. Gebiete 50 (1979), 223-236.

[8] Bingham, N.H., Goldie, C.M. and Teugels, J.L., Regular Variation, Cambridge University Press, Cambridge 1987.

[9] Comte, F., Simulation and estimation of long memory continuous time models, J. Time Series Anal. 17 (1996), 19-36.

[10] Dobrushin, R.L. and Major, P., Non-central limit theorem for the nonlinear transformations of Gaussian fields, Z. Wahrsch. Verw. Gebiete 50 (1979), 1-28.

[11] Donoghue, W.J., Distributions and Fourier Transformations, Academic Press, New York 1969.

[12] Dunford, N. and Schwartz, J.T., Linear Operator Part II, Interscience Publishing, New York 1963.

[13] Erdoğan, M.B. and Ostrovskii, I.V., Analytic and asymptotic properties of generalized Linnik probability densities, J. Math. Anal. and Appl. 217 (1998), 555-578.

[14] Fang, K.T., Kotz, S. and Ng, K.W., Symmetric Multivariate and Related Distributions, Chapman and Hall, London 1990.

[15] Gradshteyn, I.S. and Ryzhik, I.M., Table of Integrals, Series, and Products Updated Fifth Edition, Academic Press, New York 1996.

[16] Ho, H.C. and Hsing, T., Limit theorems for functionals of moving averages, Ann. Probab. 25 (1997), 1636-1669.

[17] Ivanov, A.V. and Leonenko, N.N., Statistical Analysis of Random Fields, Kluwer, Dordrecht 1989.

[18] Kotz, S., Ostrovskii, I.V. and Hayfavi, A., Analytic and asymptotic properties of Linnik probability density I \& II, J. Math. Anal. and Appl. 193 (1995), 353$371,497-521$.

[19] Leonenko, N.N., On the exactness of normal approximation of functionals of strongly correlated Gaussian random fields, Math. Notes $\mathbf{4 3}$ (1988), 283-299. (in Russian)

[20] Leonenko, N.N., Limit Theorems for Random Fields with Singular Spectrum, Kluwer, Dordrecht 1999.

[21] Leonenko, N.N. and Šilak-Benšic, M., Asymptotic properties on the LSE in a regression model with long-memory Gaussian and non-Gaussian stationary errors, Random Oper. Stoch. Eq. 4 (1996), 17-32. 
[22] Leonenko, N.N., Orsingher, E. and Parkhomenko, V.N., On the rate of convergence to the normal law for solutions of the Burgers equation with singular initial data, J. Stat. Phys. 82:3/4 (1996), 915-930. (Errata, Ibil. 84:5/6, 1389-1390).

[23] Leonenko, N.N. and Woyczynski, W.A., Exact parabolic asymptotics for singular $n$ - $D$ Burgers random fields: Gaussian approximation, Stoch. Proc. Applic. 76 (1998), 141-165.

[24] Leonenko, N.N. and Woyczynski, W.A., Scaling limits of solution of the heat equation for singular non-Gaussian data, J. Stat. Phys. 91:1/2 (1998), 423-428.

[25] Major, P., Multiple Wiener-Itô Integrals, Lecture Notes in Math 849, SpringerVerlag, Berlin 1981.

[26] McKean, H.M., Wiener's theory of nonlinear noise, In: Stoch. Diff. Eqns. Proc. SIAM-AMS 6 (1974), 191-282.

[27] Oberhettinger, F., Fourier Transform of Distributions and Their Inverse, Academic Press, New York 1973.

[28] Petrov, V.V., Limit Theorems of Probability Theory. Sequences of Independent Random Variables, Claredon Press, Oxford 1995.

[29] Plessis, N., An Introduction to Potential Theory, Oliver and Boyd, Edinburg 1970.

[30] Rosenblatt, M., Independence and dependence, In: Proc. 4th Berkeley Symp. Math. Statist. Prob., University of California Press, Berkeley (1961), 441-443.

[31] Rosenblatt, M., Some limit theorems for partial sums of quadratic form in stationary Gaussian variables, Z. Wahrsch. Verw. Gebiete 49 (1979), 125-132.

[32] Rosenblatt, M., Scale renormalization and random solutions of Burgers equation, J. Appl. Prob. 24 (1987), 328-338.

[33] Samorodnitsky, G. and Taqqu, M.S., Stable Non-Gaussian Random Processes, Chapman and Hall, New York 1994.

[34] Surgailis, D., Zones of attraction of self-similar multiple integrals, Lithuanian Math. J. 22 (1982), 327-340.

[35] Taqqu, M.S., Weak convergence to fractional Brownian motion and to the Rosenblatt process, Z. Wahrsch. Verw. Gebiete 31 (1975), 287-302.

[36] Taqqu, M.S., A representation for self-similar processes, Stoch. Proc. Appl. 7 (1978), 55-64.

[37] Taqqu, M.S., Convergence of integrated processes of arbitrary Hermite rank, $Z$. Wahrsch. Verw. Gebiete 50 (1979), 53-83.

[38] Taqqu, M.S. and Golberg, J.B., Regular multigraphs and their application to the Monte Carlo evaluation of moments of nonlinear functions of Gaussian random variables, Stoch. Proc. Appl. 13 (1982), 121-138.

[39] Viano, M.C., Deniau, C. and Oppenheim, G., Continuous-time fractional ARMA processes, Statist. and Probab. Letters 21 (1994), 323-336.

[40] Watson, G.N., A Treatise of the Theory of Bessel Functions, Cambridge University Press, Cambridge 1944. 


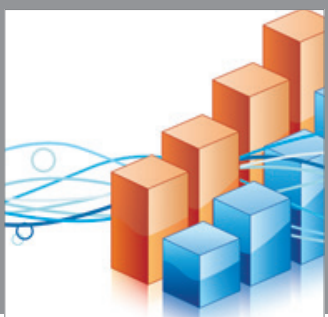

Advances in

Operations Research

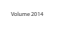

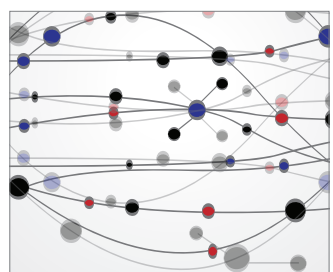

\section{The Scientific} World Journal
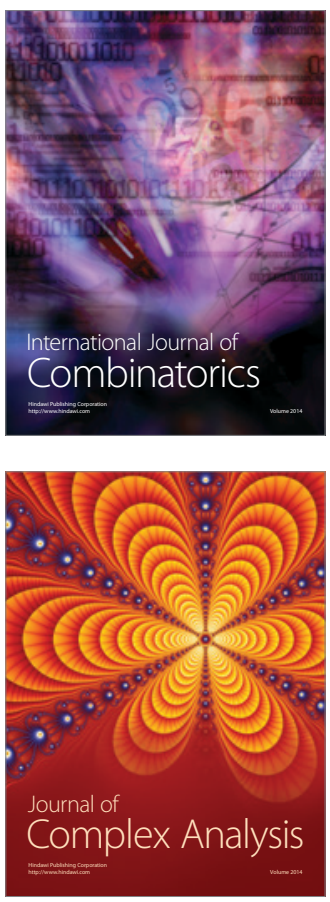

International Journal of

Mathematics and

Mathematical

Sciences
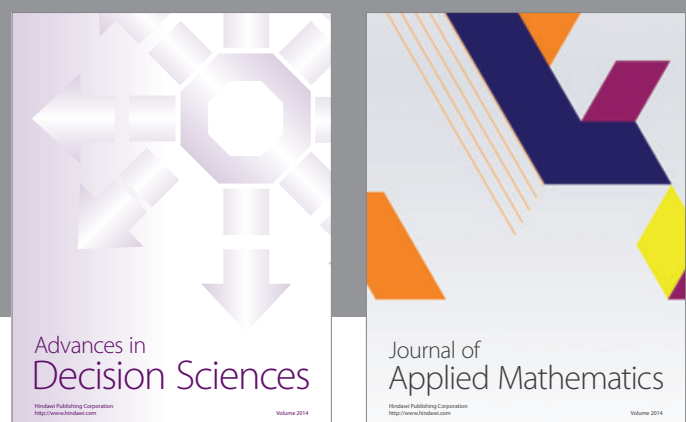

Journal of

Applied Mathematics
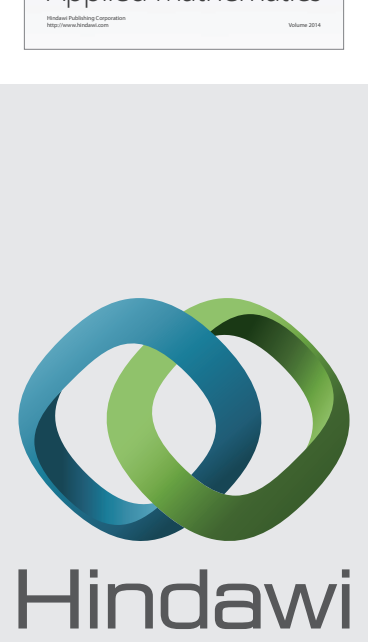

Submit your manuscripts at http://www.hindawi.com
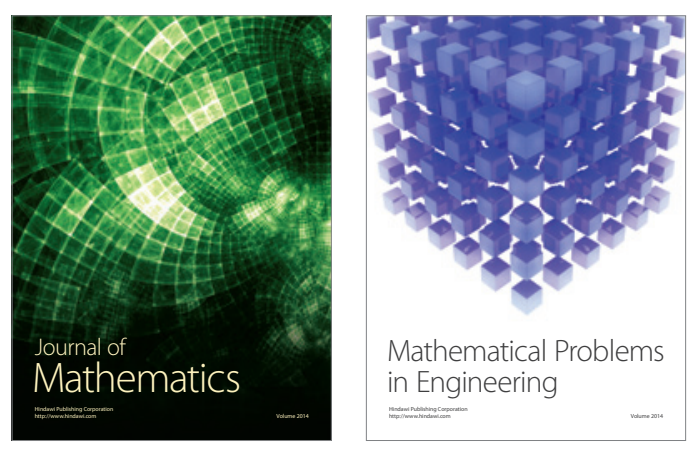

Mathematical Problems in Engineering
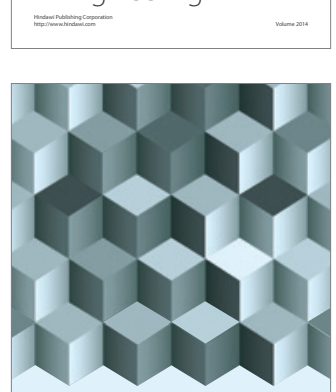

Journal of

Function Spaces
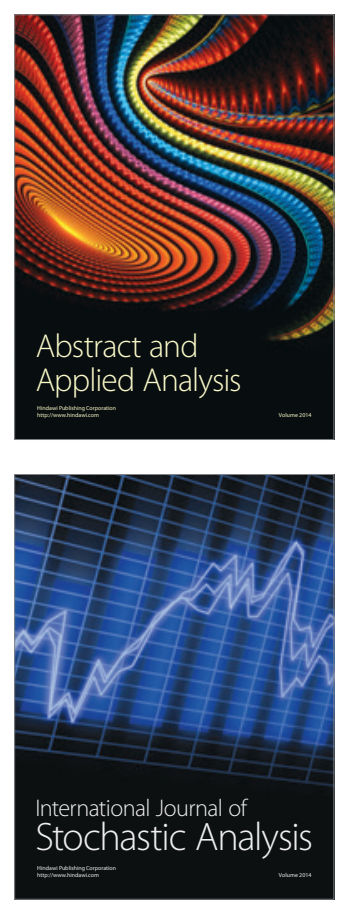

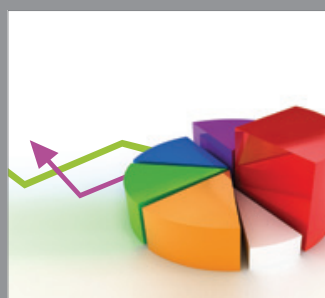

ournal of

Probability and Statistics

Promensencen
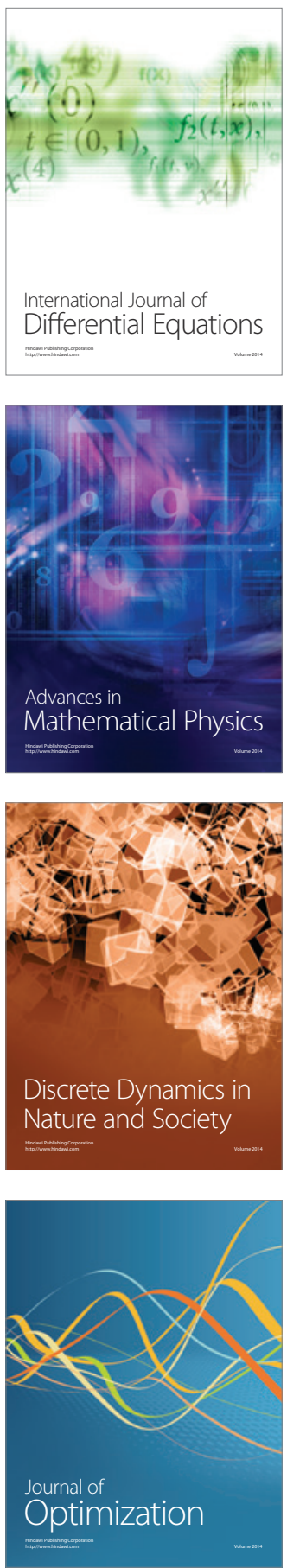